\title{
Alterstice
}

Revue internationale de la recherche interculturelle

International Journal of Intercultural Research

Revista International de la Investigacion Intercultural

\section{La migration comme métaphore, de Jean-Claude Métraux}

\section{Thomas Michaud Labonté}

Volume 2, numéro 1, 2012

URI : https://id.erudit.org/iderudit/1077558ar

DOI : https://doi.org/10.7202/1077558ar

Aller au sommaire du numéro

Éditeur(s)

Alterstice

ISSN

1923-919X (numérique)

Découvrir la revue

Citer ce compte rendu

Michaud Labonté, T. (2012). Compte rendu de [La migration comme métaphore, de Jean-Claude Métraux]. Alterstice, 2(1), 109-114.

https://doi.org/10.7202/1077558ar d'utilisation que vous pouvez consulter en ligne.

https://apropos.erudit.org/fr/usagers/politique-dutilisation/ 


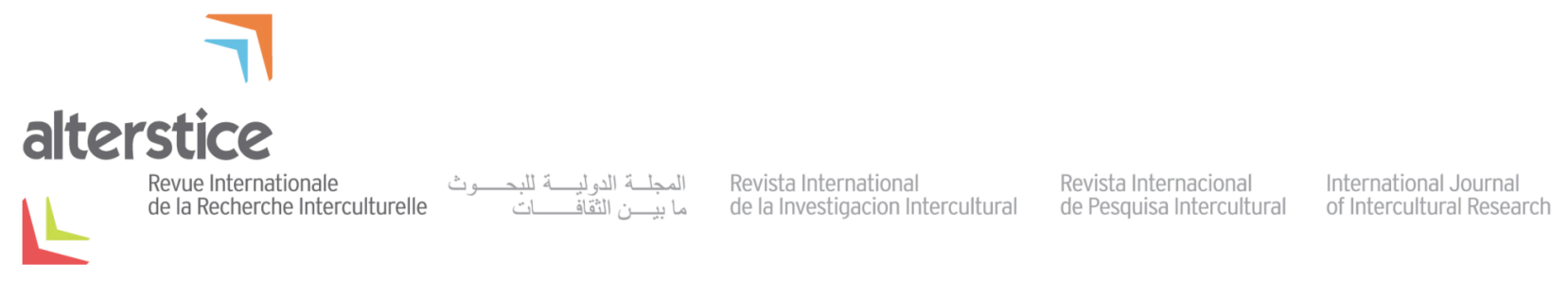

NOTE DE LECTURE

\title{
La migration comme métaphore, de Jean-Claude Métraux
}

\author{
Thomas Michaud Labonté ${ }^{1}$
}

\section{Rattachement de l'auteur \\ ${ }^{1}$ Laboratoire Psychologie et Cultures, École de Psychologie, Université Laval, Québec, Canada \\ Correspondance}

thomas.michaud-labonte.1@ulaval.ca

\section{Références de l'ouvrage :}

Métraux, J.-C. (2011). La migration comme métaphore. Paris : La Dispute.

\section{Pour citer cet article :}

Michaud Labonté, T. (2012). La migration comme métaphore [Note de lecture]. Alterstice, 2(1), $109-114$.

\section{Présentation générale}

Ce livre de 261 pages s'adresse d'abord à tous les intervenants qui œuvrent auprès de migrants. Pour l'auteur, la migration se déroule autant dans l'espace (migration géographique) que dans le temps (changements personnels et sociaux qui amènent une redéfinition des appartenances communes). Pour cette raison, on peut dire que ce livre s'adresse finalement à tous, car nul n'échappe à sa condition de migrant! L'auteur y décline la métaphore de la migration sous toutes ses formes. Dans un premier temps, il confie au lecteur des récits de vie, personnels et recueillis, qui montrent l'essence migrante de tout être, même chez les plus sédentaires. Ensuite, il propose une phénoménologie de la migration qui situe chaque étape du parcours et ses méandres. Puis l'auteur dresse un constat d'échec concernant la rencontre avec l'altérité dans les professions dites aidantes. Enfin, il élabore des pistes de solutions sous la forme de paroles précieuses et de reconnaissance.

Jean-Claude Métraux est psychiatre et psychothérapeute de l'enfant et de l'adolescent et il est aussi engagé auprès des migrants et des victimes de conflits armés. Ses parcours personnel, professionnel et politique reflètent la pluralité des voix avec laquelle il compose ses réflexions. La perspective qu'il déploie devant le lecteur est propre à enrichir le domaine de la recherche interculturelle tant par les savoirs théoriques que par les savoirs expérientiels qu'il apporte. 


\section{Ouverture}

Jean-Claude Métraux s'appuie sur le constat que les professionnels du lien, sous toutes ses formes, peinent avec l'altérité. Pour renverser cette difficulté, l'auteur annonce qu'il reprend d'abord la piste du deuil, déjà élaborée dans un ouvrage précédent (Métraux, 2004), qui aiderait nos sociétés empreintes de maîtrise à mieux rencontrer l'altérité. Or le deuil ne se commande pas. C'est pourquoi il propose aussi d'approfondir la co-construction du lien par l'entremise du don et de la reconnaissance. II vise à démontrer qu'une conception du lien social sous la forme de reconnaissance mutuelle est une thérapeutique pertinente, entre autres pour les maladies du lien que sont la précarité et l'exclusion.

Nous sommes tous des migrants, répète l'auteur, dans le temps et dans l'espace. C'est de cette façon qu'il distille l'essence d'une commune humanité pour apaiser notre obsession des différences. Et c'est aussi sur cette prémisse que le reste de son ouvrage se construit.

\section{Première partie : Nous sommes tous des migrants}

\section{Chapitre premier : Autobiographie de migrant}

Le premier chapitre est consacré à l'illustration de certains passages de l'histoire personnelle et familiale de l'auteur. Par ces récits, il met en acte les théories qui seront élaborées plus loin, en montrant que tous les processus de la migration peuvent être retrouvés dans une biographie personnelle, même chez ceux qui ne se seront pas déplacés géographiquement.

C'est ainsi qu'il aborde la vie de sa grand-mère maternelle qui, n'ayant déménagé pourtant qu'à vingt kilomètres de sa ville natale, n'a pas « réussi » son intégration à la société d'accueil. Il parle aussi de sa famille paternelle et des différences linguistiques qui sont apparues entre les générations au fil des migrations. II rapporte son admiration pour un grand-oncle qui a vécu pourtant éloigné de sa famille la majeure partie du temps. Enfin, l'auteur aborde ses propres migrations dans l'espace (Jinotepe, Sarajevo, Lausanne) et les changements de décors et de culture qui ont marqué sa jeunesse.

\section{Chapitre II : Esquisse pour une phénoménologie de la migration}

Ce chapitre élabore les moments qui se succèdent dans le processus de migration.

Vivre dans un monde et en être. Première phase du processus, nous vivons presque tous dans un ou plusieurs mondes dont chacun est créé par une communauté partageant une appartenance commune. La communauté donne du sens à son monde et s'attribue réflexivement ce sens. Il est aussi possible de vivre dans un monde sans en être, sans partager une appartenance commune, comme dans le cas des exclus et de certains migrants.

Quitter ce monde. Quitter un monde souligne la thématique du deuil qui accompagne cette phase (et les suivantes). L'élaboration du deuil se fait suivant trois phases, mais qui n'aboutissent pas toujours à un travail complet. Le refus correspond aux efforts qui sont faits pour éviter d'avoir conscience de la perte. La phase dépressive se manifeste par une conscience de la perte. Dans la dernière phase, celle du souvenir, la perte signifiante continue d'être reconnue, mais il n'y a plus de symptômes. La personne est capable de narrer de façon complexe le processus qu'elle a vécu.

Passer d'un monde à l'autre. Il s'agit ici du voyage qui s'effectue dans l'espace ou le temps, dont le départ et l'arrivée ne sont pas aisément déterminés. La durée de la transition dépend du type de frontière à franchir, du type de projet migratoire et de l'avancement du processus de deuil. Les étapes de la transition suivent celle du travail du deuil, et c'est seulement dans la phase du souvenir que le migrant se situe dans un espace transitionnel lui permettant de vivre dans un monde et d'en être.

Entrer dans un autre monde. L'entrée dans un nouveau monde débute lorsque le migrant perçoit des signes d'altérité dans le monde qui l'entoure et se termine lorsqu'il a la conviction de vivre dans un autre monde, habité 
par des appartenances différentes de la sienne. La durée de cette phase dépend de l'étendue de ses contacts et requiert que l'autre monde respecte les règles élémentaires de l’hospitalité.

Vivre dans un autre monde. Cela commence lorsque nous percevons la singularité des appartenances de l'autre monde, ce qui avive le sentiment d'étrangeté et les conflits de valeur. La durée de cette phase dépend en partie du processus de naturalisation, mais aussi de l'appartenance en tant que co-création de sens. La rencontre entre deux mondes peut amener certaines difficultés lorsqu'il y a des incompatibilités culturelles.

Être de cet autre monde. II s'agirait en principe de la dernière étape, mais celle-ci n'advient pas toujours. L'auteur revisite le modèle de Berry (1989) pour déterminer les appartenances dans la rencontre de deux mondes. Ainsi, le croisement de l'axe du sens partagé avec la communauté d'accueil (relation au présent) avec celui du sens partagé avec la communauté d'origine (relation au passé) aboutirait à quatre types d'appartenances: l'assimilation (adhérer aux valeurs de l'autre, déprécier notre propre tradition), l'intégration créatrice (reconnaissance de l'apport respectif des deux mondes), la ghettoïsation (reproduction du passé) et la double marginalisation (impossibilité de pouvoir se représenter tout avenir). Les deux derniers types d'appartenances représentent des cas où l'on peut vivre dans un monde et ne pas en être. L'auteur prévient que la simplicité de la figure est un peu réductrice, mais permet toutefois de visualiser l'enlacement des appartenances.

\section{Deuxième partie : Relents d'orientalisme}

\section{Chapitre III : De la différence au déficit}

La voie de l'orientalisme est explorée à travers un ouvrage d'Edward Said (2005) pour situer les difficultés des intervenants qui œuvrent auprès des " migrants " ${ }^{1}$. Métraux entreprend alors " un nécessaire travail de mémoire " (p. 104).

Son regard se porte d'abord sur les représentations de l'altérité menaçante en France et en Suisse ainsi que l'histoire des expansions migratoires qui eurent lieu avant 1890, année de l'apparition d'experts dans le champ de l'immigration. Ensuite, l'auteur aborde la redéfinition du Nous, passé d'un Nous communal à un Nous national, ainsi que la sélection des immigrants au niveau juridique et médical. Il discute de l'évolution des professions dans le rôle assimilateur qu'elles ont pu jouer envers les « migrants ». Enfin, il montre comment le " migrant » s'est mis à symboliser l'altérité utile jusque dans les années 1960, où ces derniers se sont trouvés plus en contact avec la société "d'accueil ». À ce moment, les experts se sont mis à traiter la différence selon une première version du modèle des déficits: "tout problème du migrant [...] s'interprète en termes de déficit vis-à-vis de la norme autochtone, et toute thérapeutique se fonde sur la présupposition d'un déficit à combler » (p. 117).

\section{Chapitre IV : Ces dix ans qui ébranlèrent nos mondes}

Jusqu'ici, l'histoire rapportée montre que la différence est hiérarchisée au profit d'une tendance sociale à la maîtrise. Le changement est pensé en termes de progrès, de croissance ou de recul. Ainsi le " migrant » est évalué de façon quantitative et est souvent accusé pour son « retard » par rapport à la société.

Métraux poursuit ainsi son récit historique dans la décennie 1970 (et début 1980). À ce moment-là, la question de la différence culturelle, prétexte aux désaccords, a alors avivé la polarité gauche/droite. Toutefois, aucun des discours citoyens de l'époque ne prêtait véritablement voix aux "migrants ". D’un côté, il y a ceux qui réclament une limitation de l'immigration, alors que de l'autre se trouvent les discours misérabilistes des sauveurs.

Les dix ans dont parle Métraux correspondent probablement à un intervalle plus ou moins défini entre le milieu des années 1980 et la fin des années 1990. On voit alors naître de multiples collaborations internationales en psychiatrie et psychothérapie, certains changements dans les pratiques institutionnelles et gouvernementales, de nouvelles pratiques en milieu scolaire, un virage dans le domaine de la recherche marqué entre autres par

\footnotetext{
${ }^{1}$ Tout comme dans le livre, l'emploi du mot « migrant » avec des guillemets réfère au sens communément utilisé.
} 
I'apparition de l'Association internationale pour le Recherche Interculturelle (ARIC) ainsi qu'un intérêt croissant pour l'interprétariat communautaire. S'il est possible de dire que la première version du modèle des déficits est ébranlée par une crise à ce moment-là, la maîtrise qui caractérise nos sociétés fait en sorte qu'un second modèle, toujours centré sur les déficits, fera apparition.

\section{Chapitre V : L'interculturalisme}

Ce chapitre passe en revue les différentes transformations qui ont eu lieu dans le passage du premier modèle des déficits vers le second. Les constats de Métraux sont les suivants :

- Cette transition a mené la notion de multiculturalisme à céder le pas à celle d'interculturalisme.

- Le programme quinquennal suisse Migration et santé (Office fédéral de la santé publique, 2006) trahit une asymétrie entre l'observateur autochtone (sujet) et le « migrant » observé (objet).

- Cinq études sont critiquées pour leur fermeture à l'altérité. Sa critique n'épargne pas l'auteur lui-même, reconnaissant par-là la difficulté de se soustraire du modèle des déficits.

- Le tiers des communications du congrès de l'ARIC en 2007 trahissent le modèle centré sur les déficits. L'étude du lien ne semble préoccuper qu'une petite proportion des congressistes (7\%).

- La thématique du traumatisme chez les " migrants » prend graduellement le dessus sur celle du deuil. Le rôle de l'intervenant est désormais de laver le traumatisme du "migrant», ce qui correspond à une résurrection du modèle des déficits.

- Deux cas de jurisprudence, qui ne sont pas des figures d'exception, montrent comment le modèle des déficits refait surface de façon claire dans le traitement de la différence culturelle en Suisse.

- En comparaison à la maladie comme métaphore, les « migrants » souffrants de maux résistants au traitement sont coupables d'être malades, d'être d'une autre culture et de ne pas avoir su comment s'intégrer à la société d'accueil.

Métraux en conclut que l'orientalisme a cédé sa place à l'interculturalisme, terme qui "tend à essentialiser les différences, à frapper les cultures du sceau de l'immuabilité » (p. 160). Dans les deux cas, l'étranger n'a pas droit à la parole. L'auteur réitère donc son constat d'échec selon lequel les «institutions et leurs représentants décortiquent le "migrant", mais ne s'interrogent guère sur leur propre épistémologie " (p. 162). L'interculturalisme ne soigne pas le migrant autant qu'il soigne le sentiment d'impuissance des intervenants.

\section{Troisième partie : Paroles précieuses}

\section{Chapitre VI : Dons de paroles}

Dans cette partie, Jean-Claude Métraux propose de revoir les modes usuels d'échanges de paroles. Selon lui, les relations entre humains impliquent trois modalités possibles. Le don favorise la co-construction et l'entretien du lien social alors que le marché et le service sont des moyens pour atteindre une fin. L'hypothèse de recherche est que les maladies du lien social (exclusion et précarité) seraient des " perturbations délétères de la dynamique du don » (p. 174).

L'auteur tente donc d'esquisser une théorie de l'échange en reprenant d'abord l'idée de Marshall Sahlins (1976) qui définit trois cercles concentriques de l'échange. Le cercle central est caractérisé par la réciprocité généralisée et est représenté par le Nous, la famille, le clan et la communauté. Le cercle médian est caractérisé par une réciprocité équilibrée et est représenté par le Vous, les personnes et les communautés alliées. Enfin, le cercle externe est caractérisé par une réciprocité inversée (s'enrichir aux dépens des autres) et est représenté par les Eux, les ennemis et les Autres.

Ensuite, Métraux applique les concepts d'objets monnaies, précieux et sacrés de Maurice Godelier (1996) aux échanges de paroles. Les paroles monnaies sont échangeables et peuvent circuler dans tous les cercles de Sahlins alors que paroles précieuses contribuent au lien d'alliance entre donateur et donataire et ne circulent que dans les 
cercles du Nous et du Vous. Les paroles sacrées, quant à elles, réfèrent à une réalité non objectivable circulant seulement dans le cercle du Nous. Les paroles peuvent changer de statut, comme une parole précieuse qui deviendrait monnaie en étant colportée d'auditoire en auditoire.

Une première solution aux problèmes du lien social consiste en l'offre de paroles précieuses. Il s'agit d'être capable de se livrer aux moments opportuns, là où notre expérience pourrait faire écho avec celle de l'interlocuteur, dans le but d'instaurer, voire restaurer, un lien de réciprocité authentique. Comme autre remède aux maladies du lien, l'auteur en appelle à la préservation des sphères sacrées en verbalisant le droit au silence des personnes qui se confient.

L'initiative du don est plus facile pour celui qui est le plus nanti, le moins menacé, et donc elle incombe aux professionnels. Mais comme donner ce qui nous est précieux est aussi comme un saut dans le vide (par rapport aux pratiques usuelles), ce n'est pas sans appréhension que nous le faisons. L'auteur propose alors une voie alternative, mais non pas de remplacement, celle de la reconnaissance.

\section{Chapitre VII : Souffles de reconnaissance}

Ce chapitre débute par un retour sur quelques formes que peuvent prendre les maladies de la reconnaissance. II situe ensuite la source du modèle des déficits transmis par les professionnels dans leur position sociale ainsi que dans les normes sociales de l'écart et de la maîtrise.

Pour soulager la souffrance sociale découlant de ces maladies, l'auteur propose une thérapeutique fondée sur le parcours de la reconnaissance de Paul Ricœur (2005). Selon ce dernier, la reconnaissance procède d'abord d'une connaissance préalable qui permet l'identification (ex. : reconnaître un ancien camarade d'école). Le parcours se poursuit vers la reconnaissance de soi-même et de ses responsabilités pour aboutir à la reconnaissance mutuelle. Métraux propose toutefois d'inverser la séquence de Ricœur afin que la reconnaissance mutuelle soit priorisée dans l'approche de l'autre par rapport à la connaissance. En ce qui concerne la connaissance de soi, le sens du parcours tel que proposé par Ricœur apparaît pertinent pour Métraux.

Ce dernier en conclut que la co-création de sens se situerait à la rencontre de la reconnaissance mutuelle de soi (aboutissement du parcours de la reconnaissance de Ricœur) et de la reconnaissance mutuelle d'autrui (premier pas du parcours de la reconnaissance de Métraux) susceptible d'engendrer une réciprocité authentique. Ainsi, la migration comme métaphore, pour soi-même et pour les autres, constitue une voie intéressante sur laquelle penser la co-construction d'une appartenance commune, migrante.

\section{Commentaires}

Par le style d'écriture qui varie abondamment entre théorie et lyrisme, la lecture de La migration comme métaphore peut paraître rebutante au lecteur fortement enculturé dans une psychologie positiviste. Pourtant, lorsqu'on arrête de lire ce livre avec l'œil de la maîtrise, on découvre que la forme de son écriture reflète l'intention même de son message. Jean-Claude Métraux exprime tout à fait la manière dont un professionnel du lien peut cocréer à partir de matériaux divers, et non pas seulement sur cette montagne croissante de recherches dites scientifiques donc sérieuses. C'est ainsi que les paroles des " migrants » qu'il a pu côtoyer prennent vie aux côtés de chercheurs et de penseurs, et toutes ces voix s'alimentent réciproquement.

Pour conclure, cet ouvrage démontre une capacité de donner vie à un propos qui aurait pu n'être qu'élucubration. Capacité de donner vie aussi dans le sens où l'auteur nous invite à sa rencontre, dévoile son intimité, et que le renouvellement de cette rencontre donne lieu, de fois en fois, à une expérience différente, toujours plus riche. 


\section{Références bibliographiques}

Berry, J. W. (1989). Acculturation et adaptation psychologique. Dans J. Retschitzki, M. Bossel-Lagos et P. Dasen (dir.), La Recherche interculturelle : tome I (p. 135-145). Paris : L'Harmattan.

Godelier, M. (1996). L'énigme du don. Paris : Fayard.

Métraux, J.-C. (2011). La migration comme métaphore. Paris : La Dispute.

Métraux, J.-C. (2004). Deuils collectifs et création sociale. Paris : La Dispute.

Ricœur, P. (2005). Parcours de la reconnaissance. Paris : Gallimard. (coll. Folio).

Said, E. W. (2005). L'orientalisme. L'orient créé par l'occident. Paris : Le Seuil.

Sahlins, M. (1976). Âge de pierre, âge d'abondance. Paris : Gallimard.

Office fédéral de la santé publique. (2002). Migration et santé : stratégie de la Confédération pour les années 2002 à 2006. Berne : Office fédéral de la santé publique. 